\title{
Real-world mepolizumab in the prospective severe asthma REALITI-A study: initial analysis
}

\author{
Tim Harrison ${ }^{1}$, Giorgio Walter Canonica $\mathbb{1}^{2,3}$, Geoffrey Chupp ${ }^{4}$, Jason Lee ${ }^{5}$, \\ Florence Schleich ${ }^{6}$, Tobias Welte $\mathbb{1}^{7}$, Antonio Valero ${ }^{8}$, Kim Gemzoe ${ }^{9}$, \\ Aoife Maxwell ${ }^{10}$, Sandra Joksaite ${ }^{11}$, Shibing Yang ${ }^{12}$, Peter Howarth ${ }^{13}$ and \\ Melissa K. Van Dyke (10 ${ }^{14}$
}

@ERSpublications

Mepolizumab has demonstrated efficacy in patients with severe eosinophilic asthma in the controlled environment of clinical trials. These initial data from the prospective REALITI-A study show that similar results are obtained in a real-world setting. https://bit.ly/3hINnFO

Cite this article as: Harrison T, Canonica GW, Chupp G, et al. Real-world mepolizumab in the prospective severe asthma REALITI-A study: initial analysis. Eur Respir J 2020; 56: 2000151 [https://doi.org/10.1183/ 13993003.00151-2020].

\section{ABSTRACT}

Introduction: Efficacy of mepolizumab, an anti-interleukin-5 monoclonal antibody, was demonstrated in randomised controlled trials; data on its real-world impact in routine clinical practice are starting to emerge. We assessed the effectiveness and safety of mepolizumab prescribed for patients in the real world. Methods: REALITI-A is a global, prospective, observational cohort study, collecting data from routine healthcare visits from patients with asthma. Patients newly prescribed mepolizumab for severe asthma with 12 months of relevant medical history pre-mepolizumab (collected retrospectively) were enrolled. An initial analysis of data from early initiators who had completed 1 year of follow-up (as of February 28, 2019) was conducted. The primary objective was to compare the rate of clinically significant exacerbations (requiring oral corticosteroids (OCS) and/or hospitalisation and/or emergency department visit) before and after mepolizumab; exacerbations requiring hospitalisation and/or emergency department visit and change in maintenance OCS use were secondary objectives. Treatment-related adverse events were reported.

Results: Overall, 368 mepolizumab-treated patients were included. Rates of clinically significant exacerbations were reduced by $69 \%$ from 4.63 per person per year pre-treatment to 1.43 per person per year during follow-up $(\mathrm{p}<0.001)$, as were those requiring hospitalisation and/or emergency department visit (from 1.14 to 0.27 per person per year; 77\% reduction). In 159 patients with maintenance OCS dose data available during the pre-treatment period, median daily dose decreased from 10.0 (pre-treatment) to $5.0 \mathrm{mg} \cdot \mathrm{day}^{-1}$ by week 21-24 of follow-up, sustained until week 53-56. No new safety signals were reported.

Conclusion: These data demonstrate that the effectiveness of mepolizumab is consistent with clinical trial results under real-world settings, with significant reductions in exacerbations and daily maintenance OCS dose.

This article has supplementary material available from erj.ersjournals.com

Data sharing: Upon completion of the primary analysis, anonymised individual participant data and study documents can be requested for further research from www.clinicalstudydatarequest.com.

Received: 23 Jan 2020 | Accepted after revision: 23 July 2020

Copyright $\odot$ ERS 2020. This article is open access and distributed under the terms of the Creative Commons Attribution Non-Commercial Licence 4.0. 


\section{Introduction}

Of the estimated 300 million people worldwide with asthma, 5-10\% are expected to experience severe disease, placing a significant burden on patients and healthcare systems [1-6]. Many patients with severe asthma receiving maximal inhaled controller medication continue to experience exacerbations [1]. Severe eosinophilic asthma is one of several phenotypes of severe asthma, and is associated with persistent eosinophilic inflammation, reduced lung function, poor asthma control and recurrent exacerbations, with/ without systemic corticosteroid (SCS) use [1,7-11].

Mepolizumab is an anti-interleukin-5 monoclonal antibody that selectively inhibits eosinophilic inflammation [12]. In clinical trials, add-on mepolizumab therapy, to standard of care, reduced exacerbations, decreased oral corticosteroid (OCS) dependence, and improved lung function, asthma control and health-related quality of life versus matched placebo in patients with severe eosinophilic asthma with a history of exacerbations [13-16]. It is approved as an add-on treatment for patients with severe eosinophilic asthma $[17,18]$.

Clinical trial eligibility criteria often result in a more homogenous patient population regarding demographics and disease characteristics than patients treated in routine clinical practice $[8,19]$. Although clinical trials have high internal validity, they do not replicate real-world conditions [20]. Indeed, a manifesto by the Respiratory Effectiveness Group stated it is necessary to obtain data on outcomes from patients treated in the real world for external validity, to complement clinical trials and guide treatment-related decisions [21]. The 24-month REALITI-A study evaluates mepolizumab use in clinical practice. Here, we report an initial analysis of data from patients who had completed 12 months of follow-up by February 28, 2019, following mepolizumab initiation. They represent some of the first to be prescribed mepolizumab in real-world clinical practice.

\section{Methods}

Subjects

Eligible patients were aged $\geqslant 18$ years with a current clinical diagnosis of asthma, a physician decision to initiate mepolizumab treatment and relevant medical records for $\geqslant 12$ months pre-enrolment, and who had provided informed consent for study participation. Prior use of other biological medications was permitted; those who had received mepolizumab in the year pre-enrolment were excluded. Patients who had participated in an interventional clinical trial within the year pre-enrolment were also excluded. Patients were recruited from 51 centres in seven countries (table 1).

\section{Study design}

REALITI-A (GSK study identifier 204710) is a global, prospective, single-arm, observational cohort study enrolling patients diagnosed with asthma and newly prescribed mepolizumab treatment (physician decision) (figure 1 and supplementary material). The index date was the first mepolizumab administration. Enrolment could occur before or after the index date; if occurring before the index date, there was a variable-length run-in period (driven by local prescribing and dispensing practices) between the enrolment and index dates, where the same therapy was continued. There was no run-in period when the enrolment and index dates were the same day or when enrolment occurred after the index date (maximum 7 days permitted from index to enrolment).

The pre-mepolizumab treatment period ended on the index date and started: 1) 365 days pre-enrolment date if the index date was before the enrolment date; 2) 365 days (+1 day) if the index date and enrolment

Affiliations: ${ }^{1}$ Nottingham NIHR Biomedical Research Centre, Nottingham City Hospital, University of Nottingham, Nottingham, UK. ${ }^{2}$ Personalized Medicine Asthma and Allergy Clinic, Dept of Biomedical Sciences, Humanitas University and Research Hospital, Rozzano, Milan, Italy. ${ }^{3}$ Dept of Biomedical Science, Humanitas University, Pieve Emanuele, Milan, Italy. ${ }^{4}$ Division of Pulmonary, Critical Care, and Sleep Medicine, Dept of Internal Medicine, Yale School of Medicine, New Haven, CT, USA. ${ }^{5}$ Toronto Allergy and Asthma Clinic, Toronto, ON, Canada. ${ }^{6}$ Dept of Pulmonary Medicine, CHU Sart-Tilman and GIGA-I3 Research Group, University of Liège, Liège, Belgium. ${ }^{7}$ Dept of Respiratory Medicine and German Center for Lung Research, Hannover Medical School, Hannover, Germany. ${ }^{8}$ Sección de Alergología, Servicio de Neumología y Alergia, Hospital Clínic de Barcelona, Universitat de Barcelona, IDIBAPS, CIBER de Enfermedades Respiratorias (CIBERES), Barcelona, Spain. ${ }^{9}$ Real World Study Delivery, Value Evidence and Outcomes, Global Medical, GSK, Uxbridge, UK

${ }^{10}$ Real World Study Delivery, Value Evidence and Outcomes, Global Medical, GSK, Stevenage, UK. ${ }^{11}$ Clinical Statistics, R\&D Projects Clinical Platforms and Sciences, GSK, Uxbridge, UK. ${ }^{12}$ Value Evidence and Outcomes, GSK, Collegeville, PA, USA. ${ }^{13}$ Global Specialty \& Primary Care, GSK, Brentford, UK. ${ }^{14}$ Epidemiology, GSK, Upper Providence, PA, USA.

Correspondence: Peter Howarth, Global Specialty \& Primary Care, GSK, GSK House, 980 Great West Road, Brentford, Middlesex, TW8 9GS, UK. E-mail: peter.h.howarthdggsk.com 
TABLE 1 Demographics and clinical characteristics during the pre-mepolizumab treatment period (baseline)

Total population

Age at enrolment years $(n=367)$

Mean \pm SD

$53.1 \pm 13.7$

Median (IQR)

Female (n=367)

$4.0(44.0-63.0)$

Race

White/Caucasian

Asian, Native Hawaiian or other Pacific Islander

$226(62)$

Black/African-American

$337(92)$

$17(5)$

$5(1)$

Other/multiple

9 (2)

Country

UK

136 (37)

Italy

87 (24)

Germany

44 (12)

Canada

$40(11)$

Belgium

29 (8)

Spain

24 (7)

USA

8 (2)

BMI at enrolment $\mathrm{kg} \cdot \mathrm{m}^{-2}$

Mean \pm sD

$28.7 \pm 7.3$

Median (IQR)

Smoking history at enrolment ( $n=364)$

$221(61)$

Never-smoker

$10(3)$

Current smoker

Ex-smoker

133 (37)

Asthma duration at enrolment years $(n=360)$

Mean \pm SD

Median (IQR)

Previous use of omalizumab $(n=365)$

Duration of omalizumab treatment months $(\mathbf{n}=\mathbf{7 0})$

Mean \pm SD

Median (IQR)

$29.1 \pm 31.7$

Maintenance OCS use at enrolment ${ }^{\pi}(n=365)$

Never

$14.5(6.0-48.0)$

Past

$108(30)$

83 (23)

Current

$174(48)$

Daily maintenance $0 \mathrm{CS}$ dose ${ }^{+} \mathrm{mg} \cdot \mathrm{day}^{-1}(\mathrm{n}=159)$

Mean \pm SD

Median (IQR)

$14.4 \pm 19.5$

Rate of exacerbations per person per year $(n=366)$

$10.0(5.0-15.0)$

Clinically significant exacerbations

Mean \pm SD

Median (IQR)

Exacerbations requiring hospitalisation and/or emergency department visit

Mean \pm sD

Median (IQR)

$4.63 \pm 4.09$

$3.0(2.0-6.0)$

Exacerbations

$1.14 \pm 2.26$

$0(0.0-1.0)$

Mean \pm SD

$0.60 \pm 1.33$

Median (IQR)

Blood eosinophil count ${ }^{\S}$ cells $\cdot \mu L^{-1}(n=357)$

Geometric mean \pm sD logs

Median (IQR)

$0(0.0-1.0)$

$<150$

$150-<300$

2 (270-800)

51 (14)

45 (13)

$\geqslant 300$

261 (73)

Lung function ${ }^{\S}$

Pre-bronchodilator $\mathrm{FEV}_{1} \mathrm{~L}(\mathrm{n}=201)$

Mean \pm SD

$1.94 \pm 0.79$

Median (IQR)

$1.84(1.39-2.42)$

Pre-bronchodilator FVC L $(n=201)$

Mean \pm SD

$2.93 \pm 1.02$

Median (IQR)

$2.82(2.28-3.57)$ 


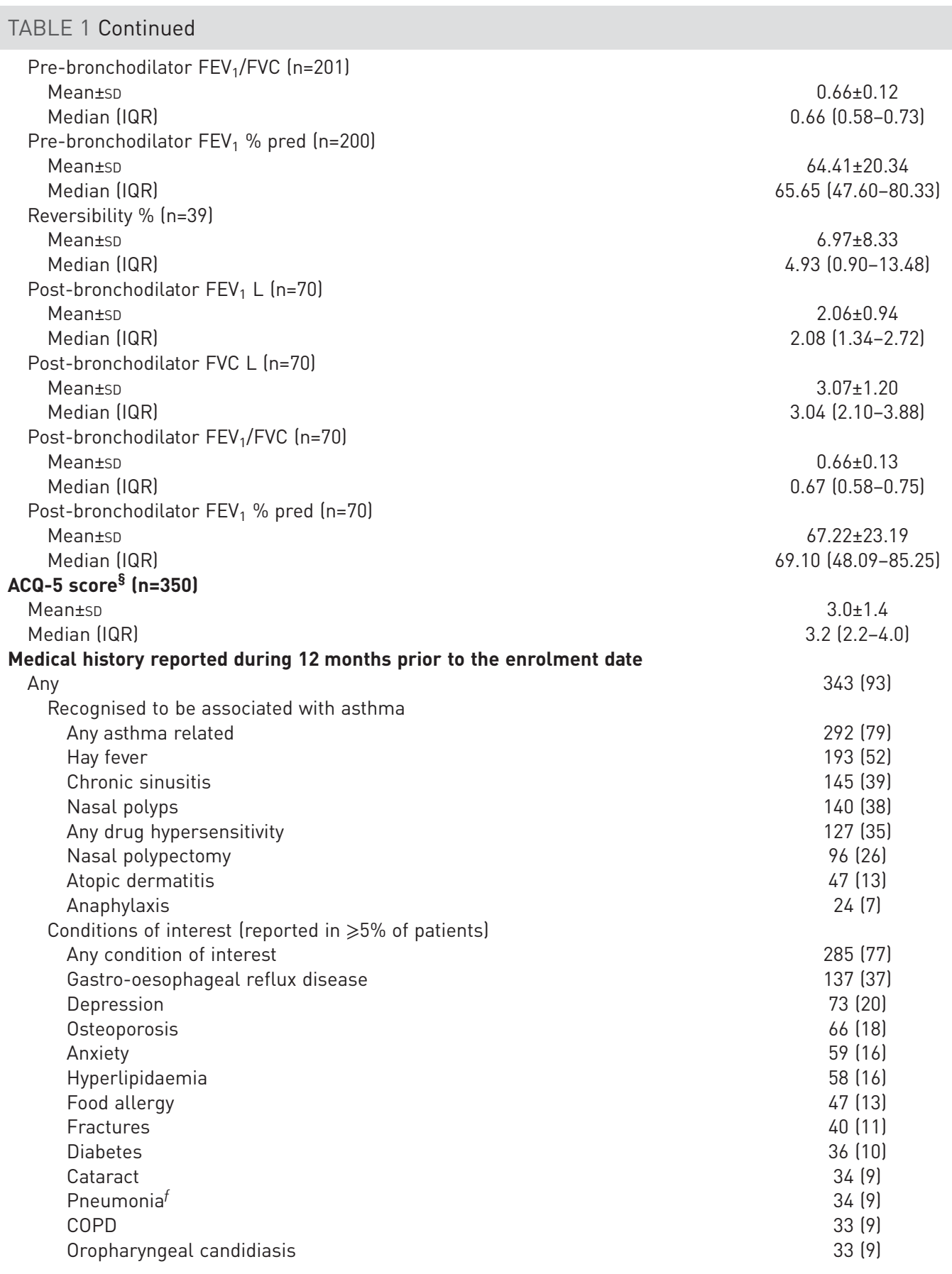

Data are presented as $\mathrm{n}$ or $\mathrm{n}(\%)$, unless otherwise stated; percentages may not add up to $100 \%$ due to rounding. IQR: interquartile range; BMI: body mass index; OCS: oral corticosteroid; $\mathrm{FEV}_{1}$ : forced expiratory volume in $1 \mathrm{~s}$; FVC: forced vital capacity; ACQ-5: five-item Asthma Control Questionnaire; COPD: chronic obstructive pulmonary disease. " : during the enrolment period, 13 out of $71(18 \%)$ patients had received omalizumab treatment outside of a clinical trial and the remaining 58 out of 71 (82\%) patients had received omalizumab treatment $>12$ months prior to enrolment that may have been within or outside of a clinical trial (data were collected for 12 months prior to enrolment only); in those patients who received omalizumab in the prior 12 months, typically, the end of omalizumab treatment was $~ 1-2$ months prior to starting mepolizumab. ๆ: reported by the patient. ${ }^{+}$: during the period including the index date and the 27 days prior to index or any other 27-day period in the last 6 months if no records existed 27 days immediately prior to index. ${ }^{\S}$ : latest record prior to index. ${ }^{f}$ : pneumonia cases were limited to the prior 12 months. 
date were the same or 3) 365 days (+run-in period+1 day) if the enrolment date was pre-index date. Data were collected retrospectively at enrolment (and, if relevant, prospectively during the run-in period) from medical records and patient recall for the previous year.

The 12-month follow-up period after initiating mepolizumab was from the index date $(+1$ day) to the first of: death, withdrawal of consent or end of follow-up. The present analysis was conducted in the first cohort of participants in the study who, on February 28, 2019, had completed 12 months of follow-up after starting mepolizumab. Data were collected prospectively at asthma healthcare visits (routine or unscheduled) during the 12-month post-index period; no visits were scheduled specifically for this observational study. The study population subset included in this analysis was enrolled from December 2016 to February 2018; full study population enrolment completed on October 31, 2019.

\section{Outcomes}

The primary objective was to compare the clinically significant asthma exacerbation rate in the pre-mepolizumab treatment period versus the 12-month follow-up period. Clinically significant exacerbations were defined as a deterioration in asthma requiring SCS (any dose; oral steroids (e.g. prednisone) for $\geqslant 3$ days or a single systemic administration of corticosteroids (intravenous/intramuscular dose)) and/or hospitalisation and/or emergency department visit. For patients already receiving maintenance SCS, at least double the existing maintenance dose for $\geqslant 3$ days was required. Exacerbations treated with courses of corticosteroids separated by $<7$ days were classed as the same exacerbation (based on data entry by physicians).

Secondary objectives compared the following outcomes in the same periods: asthma exacerbation rates requiring hospitalisation and/or emergency department visit, or hospitalisation only, and proportion of patients without clinically significant exacerbations, and those achieving $>0-<50 \%$ and $\geqslant 50-100 \%$ reductions in clinically significant exacerbation rates. Additionally, for patients who reported at enrolment they were receiving maintenance OCS (supplementary material), the change and percentage reduction in median daily maintenance OCS dose from pre-treatment to 12 months after initiating mepolizumab were assessed. The proportion of patients receiving maintenance OCS pre-mepolizumab treatment who discontinued maintenance OCS with mepolizumab was also reported. Change from baseline in blood eosinophil count was also assessed. Safety objectives included reported mepolizumab-related adverse events and serious adverse events. The relationship of adverse events to mepolizumab was determined by the investigator without further adjudication.

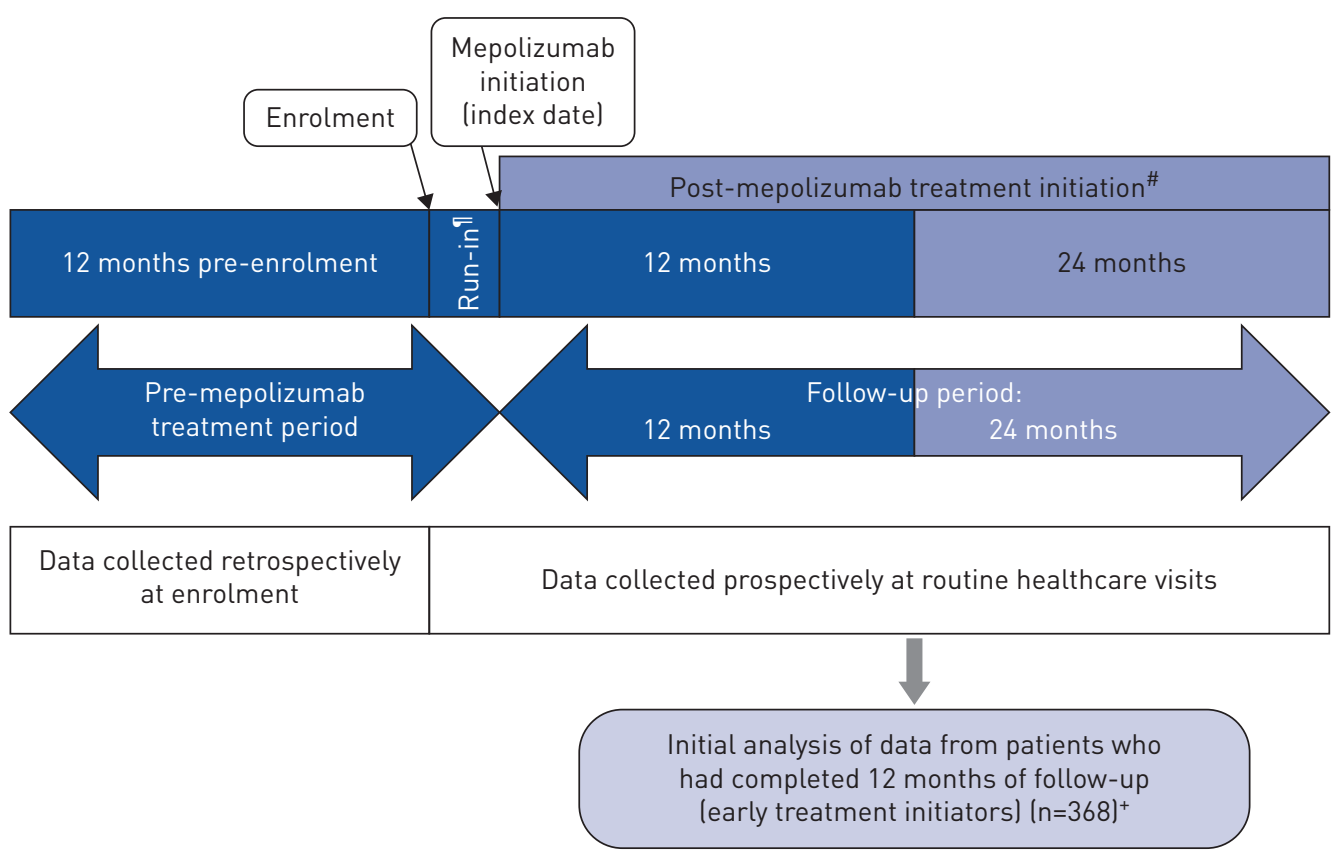

FIGURE 1 Study design. *: there will be a 12-month interim analysis of the full study population (primary and secondary objectives) and a 24-month analysis of the full study population (secondary objectives). ": if enrolment occurred before the index date, there was a variable-length run-in period where patients continued with the same therapy; there was no run-in period when the enrolment and index dates were the same day or when the index date occurred before enrolment. ${ }^{+}$: data cut-off 28 February 2019. 


\section{Analysis}

With the assumption that $25 \%$ of patients would withdraw during the full study period (supplementary material), a 12-month mepolizumab treatment period with 200 patients was expected to have $90 \%$ power to detect a $35 \%$ reduction in clinically significant exacerbations at the two-sided 5\% level. The treated population, used for all effectiveness and safety evaluations, included all enrolled patients who received one mepolizumab dose or more. Subgroup analyses were performed by blood eosinophil count $(<150, \geqslant 150-$ $<300$ and $\geqslant 300$ cells $\mu \mathrm{L}^{-1}$ ) at index or the most recent count available pre-index. Additional post hoc subgroup analyses for clinically significant exacerbations were performed by maintenance OCS use and dose in the pre-treatment period (yes versus no; $<10$ versus $\left.\geqslant 10 \mathrm{mg} \cdot \mathrm{day}^{-1}\right)$, age at enrolment $(<65$ versus $\geqslant 65$ years of age) and prior omalizumab use during lifetime (yes versus no), and for blood eosinophil count by maintenance OCS use and dose in the pre-treatment period.

A treatment policy estimand approach for treatment discontinuation was used in this study, which provided an estimate of the expected effect of mepolizumab using all data collected during the 12-month follow-up period, regardless of whether patients discontinued mepolizumab. This analysis corresponds to an intent-to-treat analysis in a clinical trial.

The exacerbation rate in the pre-treatment and 12-month follow-up periods was analysed using negative binomial regression with time period (pre-treatment and 12-month follow-up) as a covariate. The mean estimate variance was corrected for within-patient correlation by use of generalised estimating equations (GEEs). The proportion of patients without clinically significant exacerbations was analysed using logistic regression and pre-treatment versus post-treatment initiation period data were compared via GEEs with time period as a covariate. Proportions of patients with $\geqslant 50 \%$ reduction in clinically significant exacerbation rates are also reported. The mean OCS dose was calculated for each patient over each 28-day period during pre-treatment and the 12-month follow-up. For the mean calculation, a $0 \mathrm{mg}$ dose was assumed if no data were recorded for a specific day (supplementary material); if a recorded dose could not be interpreted, the mean was based on the number of days with interpretable data. Summary statistics (including median) of the OCS maintenance dose were based on the mean value calculated as described earlier. Patient-specific percentage change from baseline in maintenance OCS daily dose was calculated at each post-treatment time-point. Applying the distribution-free method [22] to this variable, the median percent reduction (with 95\% confidence interval) from baseline was estimated at 12 months post-treatment initiation (i.e. week 53-56). The ratio to baseline for blood eosinophil count was assessed using mixed model repeated measures. Only data on mepolizumab-related adverse events or other GSK products were collected during the study.

This study was conducted in accordance with the Declaration of Helsinki. Local ethical approval was obtained per study site.

\section{Results}

\section{Patients}

Overall, 368 patients received one mepolizumab dose or more in the treated population. The UK enrolled the most patients $(n=136)$ in this analysis (table 1$)$. The mean body mass index at enrolment was $28.7 \mathrm{~kg} \cdot \mathrm{m}^{-2}, 39 \%$ (143 out of 364 ) of patients were current smokers or ex-smokers and $48 \%$ (174 out of 365) were receiving maintenance OCS pre-mepolizumab treatment (table 1). Of those with baseline blood eosinophil count data available, 51 out of $357(14 \%)$ had baseline blood eosinophil counts $<150$ cells. $\mu \mathrm{L}^{-1}$, 45 out of 357 (13\%) had counts $\geqslant 150-<300$ cells $\mu \mathrm{L}^{-1}$ and 261 out of $357(73 \%)$ had counts $\geqslant 300$ cells $\mu \mathrm{L}^{-1}$. When stratified by baseline blood eosinophil count, 32 out of $51(63 \%), 32$ out of 45 $(71 \%)$ and 108 out of $258(42 \%)$ patients in the $<150, \geqslant 150-<300$ and $\geqslant 300$ cells. $\mu \mathrm{L}^{-1}$ subgroups, respectively, reported using maintenance OCS in the pre-treatment period. Additionally, 38\% (140 out of 368) of patients had a clinical history of nasal polyps.

At data cut-off, patients had received a mean \pm SD of $11.4 \pm 3.11$ mepolizumab treatments, with a mean \pm SD treatment duration of $340.4 \pm 87.24$ days. Overall, 70 out of $368(19 \%)$ patients discontinued mepolizumab during the 12-month follow-up; the most common reason was participant decision (27 out of 368 (7\%)), while 13 out of 368 (4\%) patients reported lack of efficacy (supplementary table S1).

\section{Exacerbations}

The clinically significant exacerbation rate fell from 4.63 per person per year in the pre-mepolizumab treatment period to 1.43 per person per year in the 12-month follow-up, equating to a significant $69 \%$ reduction (rate ratio 0.31 (95\% CI 0.27-0.35); p<0.001) (figure 2). Exacerbation rate reductions were similar across baseline blood eosinophil count subgroups (figure 2). 


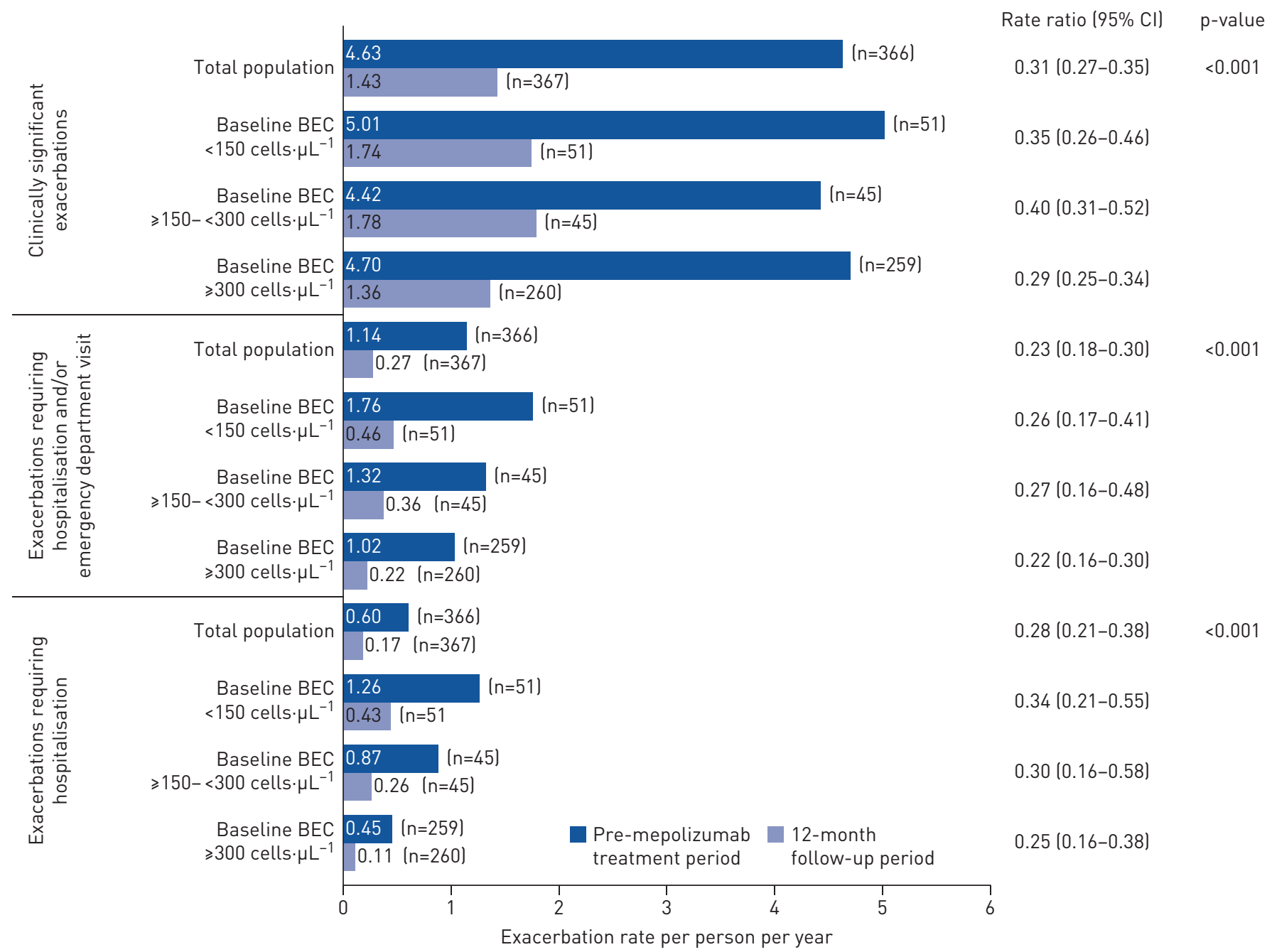

FIGURE 2 Asthma exacerbation rates in the pre-mepolizumab treatment period $(365$ days prior to enrolment plus any exacerbations starting during run-in) and 12-month follow-up period. BEC: blood eosinophil count. The $n$-value indicates the number of patients.

The exacerbation rate requiring hospitalisation and/or emergency department visit was also significantly reduced by $77 \%$ from 1.14 per person per year pre-treatment to 0.27 per person per year during follow-up (rate ratio 0.23 (95\% CI 0.18-0.30); $<<0.001$ ); reductions were observed across all baseline blood eosinophil count subgroups (figure 2). Additionally, there was a significant reduction $(p<0.001)$ in the exacerbation rate requiring hospitalisation in the overall population following treatment, with a similar trend observed across all baseline blood eosinophil count subgroups.

Overall, 67\% (247 out of 366 ) of patients achieved a $\geqslant 50 \%$ reduction in clinically significant exacerbations from pre-treatment to 12 months after mepolizumab initiation; this rose to $73 \%$ (247 out of 340 ) when those without a prior history of exacerbations pre-treatment were excluded (table 2). Furthermore, a significantly higher proportion of patients had no clinically significant exacerbations during follow-up (48\%) versus pre-treatment (7\%; OR 12.13 (95\% CI 8.03-18.33); $\mathrm{p}<0.001$ ) (figure 3). Across baseline blood eosinophil count subgroups, the proportion of patients without clinically significant exacerbations during follow-up rose to $38-49 \%$ versus $7-9 \%$ during pre-treatment (figure 3). Clinically significant exacerbations assessed by maintenance OCS use and dose in the pre-treatment period, age at enrolment, and prior omalizumab use are shown in supplementary figure $\mathrm{S} 1$.

\section{Maintenance OCS}

Data on maintenance OCS dose during pre-treatment were available for 159 patients (supplementary material). The median (interquartile range (IQR)) daily maintenance OCS dose fell from 10.0 (5.015.0) $\mathrm{mg}$ day $^{-1}$ during pre-treatment to $5.0(0.9-10.0) \mathrm{mg}^{-}$day $^{-1}$ by week $21-24$ and remained at the same level until week 53-56 (5.0 (0.0-7.5) mg.day $\left.{ }^{-1}\right)$. The corresponding median percent reduction was $52 \%$ 
TABLE 2 Patients achieving a reduction ${ }^{\#}$ in the rate of clinically significant exacerbations during the 12-month follow-up period

\begin{tabular}{lcc} 
& $\begin{array}{c}\text { Total } \\
\text { population }\end{array}$ & $\begin{array}{c}\text { Population with one exacerbation or more } \\
\text { in the pre-mepolizumab treatment period }\end{array}$ \\
\hline Subjects & 368 & 340 \\
$\geqslant \mathbf{5 0 - 1 0 0 \% \text { reduction }}$ & $247(67)$ & $247(73)$ \\
$>\mathbf{0}-<\mathbf{5 0 \%}$ reduction & $52(14)$ & $52(15)$ \\
No change/increase & $66(18)$ & $40(12)$ \\
Missing & $1(<1)$ & $1<1)$
\end{tabular}

Data are presented as $\mathrm{n}$ or $\mathrm{n}(\%) .{ }^{*}$ : based on 365 days prior to enrolment plus any exacerbations starting during run-in; ": denominator based on patients with data for historical exacerbations only ( $n=366$ ); ${ }^{+}$: denominator based on patients with one historical exacerbation or more only $(n=340)$; patients with zero historical exacerbations were excluded $(n=26) ;{ }^{\S}$ : one patient did not have data during the follow-up period and is therefore not included in any of the categories.

(95\% CI 50.0-75.0\%) (i.e. week 53-56) (figure 4a). Of the 125 patients on maintenance OCS at week 5356 with data available, 82 remained on OCS at week 53-56, with $34 \%$ (43 out of 125) of patients discontinuing OCS while on mepolizumab (data are from the while-on-treatment estimand for treatment discontinuation) (figure 5).

In the $<150, \geqslant 150-<300$ and $\geqslant 300$ cells $\mu \mathrm{L}^{-1}$ baseline blood eosinophil count subgroups, the median daily maintenance OCS dose during pre-treatment was reduced from 12.8, 11.3 and 9.8 to 6.5, 5.0 and $2.5 \mathrm{mg} \cdot \mathrm{day}^{-1}$, respectively, by week 53-56; reductions were seen as early as week $9-12,13-16$ and 5-8, respectively (figure $4 \mathrm{~b}-\mathrm{d}$ ). At week $53-56$, median percent reductions from baseline in median daily maintenance OCS dose in the $<150, \geqslant 150-<300$ and $\geqslant 300$ cells $\mu \mathrm{L}^{-1}$ baseline blood eosinophil count subgroups were $51 \%$ (95\% CI 22-74\%), 23\% (95\% CI 0-69\%) and 74\% (95\% CI 50-100\%), respectively.

\section{Blood eosinophil count}

Following mepolizumab treatment initiation, blood eosinophil count was reduced from least squares geometric mean $370(95 \%$ CI $320-410)$ cells $\mu \mathrm{L}^{-1}$ at baseline to $60(95 \%$ CI $50-80)$ cells $\mu \mathrm{L}^{-1}$ at month

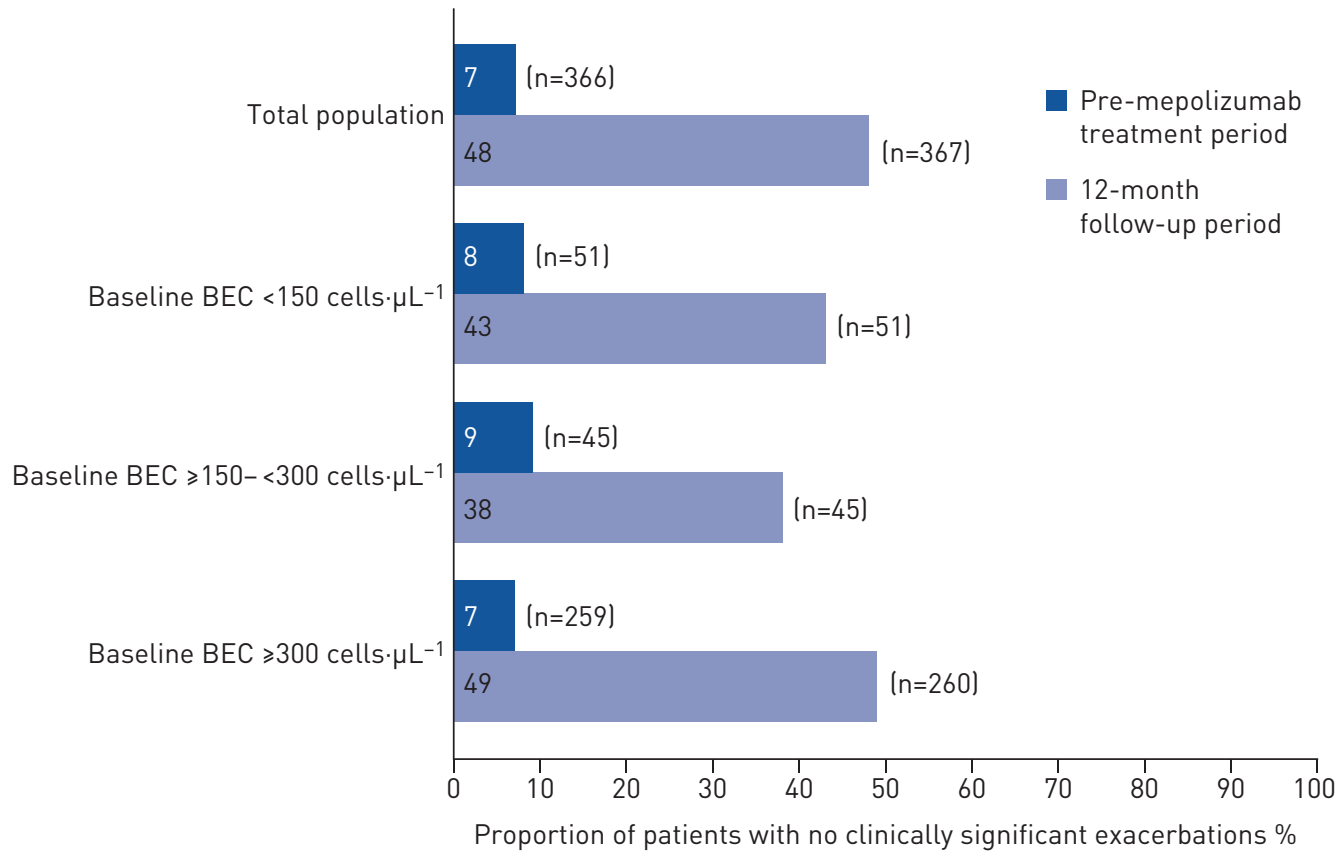

FIGURE 3 Proportion of patients with no clinically significant exacerbations in the pre-mepolizumab treatment period (365 days prior to enrolment plus any exacerbations starting during run-in) and 12-month follow-up period. BEC: blood eosinophil count. The $n$-value indicates the number of patients. 


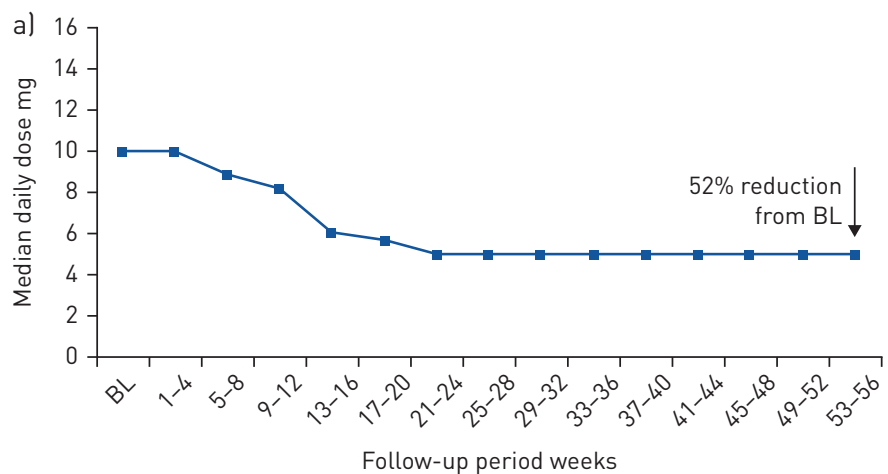

n: $159158156156 \quad 154153153152151150149146143 \quad 143 \quad 143$

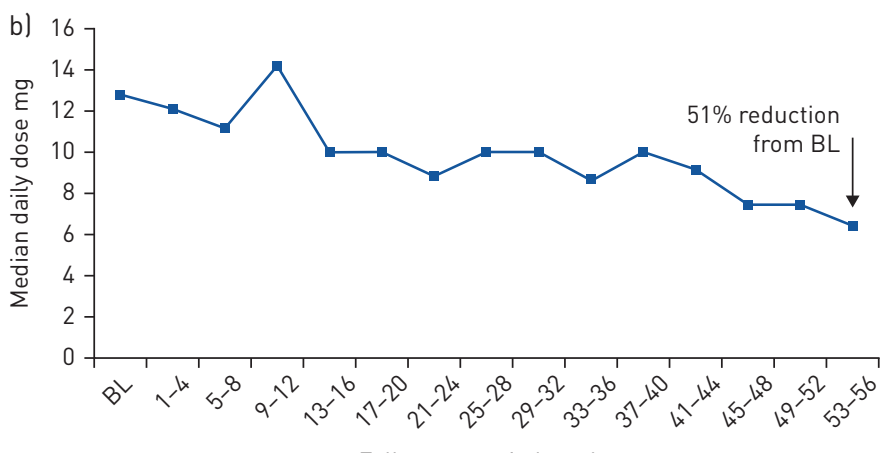

Follow-up period weeks

n: $\quad \begin{array}{lllllllllllllll}28 & 28 & 28 & 28 & 28 & 27 & 27 & 27 & 27 & 27 & 27 & 26 & 24 & 24 & 24\end{array}$
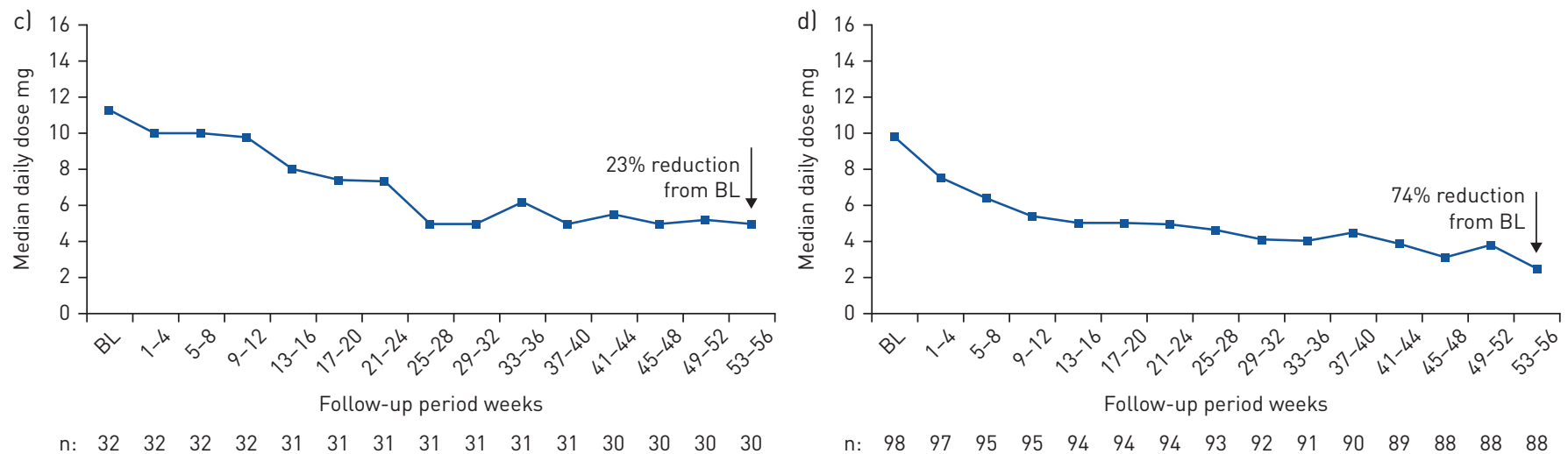

FIGURE 4 Maintenance oral corticosteroid (OCS) use after initiation with mepolizumab treatment for a) the overall population and according to the following baseline (BL) blood eosinophil count subgroups: b) $<150, c) \geqslant 150-<300$ and d) $\geqslant 300$ cells. $\mu L^{-1}$. The median percentage change was calculated using the distribution-free method [22] with patient-specific percentage change from $B L$ as a variable. The $n$-value indicates the number of patients.

9-12 (median (IQR) blood eosinophil count values were $442(270-800)$ cells $\mu \mathrm{L}^{-1}$ at baseline and $90(40-$ 100) cells $\mu \mathrm{L}^{-1}$ at month $9-12$ ). This corresponds to a reduction of $83 \%$ to 60 cells $\mu \mathrm{L}^{-1}$ (least squares mean ratio to baseline at month 9-12: 0.17 (95\% CI 0.13-0.21)). The reduction in blood eosinophil count was observed by month $0-3$ and was maintained throughout the 12-month follow-up. Supplementary table S2 presents blood eosinophil count by maintenance OCS use and dose in the pre-treatment period.

\section{Safety}

Overall, 53 out of 368 (14\%) patients experienced an investigator-determined treatment-related adverse event during follow-up (table 3 ). The most common adverse events (occurring in $\geqslant 2 \%$ of patients) were disorders classified as affecting the nervous system (predominantly headache), general and administration site, musculoskeletal and connective tissue, skin and subcutaneous tissue, and gastrointestinal tract. Treatment-related serious adverse events were experienced by two out of $368(<1 \%)$ patients during follow-up (table 3). Treatment-related serious adverse events of hypersensitivity and pharyngeal swelling were experienced by one patient each. During follow-up, nine out of 368 (2\%) patients experienced a treatment-related adverse event, leading to permanent treatment discontinuation (table 3). No treatment-related deaths occurred.

\section{Discussion}

The REALITI-A study is a prospective, global, observational, self-controlled cohort study being conducted to collect real-world data from patients with asthma who were newly prescribed mepolizumab treatment. These initial results showed real-world mepolizumab initiation led to significant reductions in the annual asthma exacerbation rate and clinically meaningful reductions in daily maintenance OCS dose versus pre-mepolizumab treatment. Furthermore, there were no new safety concerns with mepolizumab when compared with results from previous randomised controlled trials (RCTs). These initial data confirm mepolizumab effectiveness in a real-world setting. 


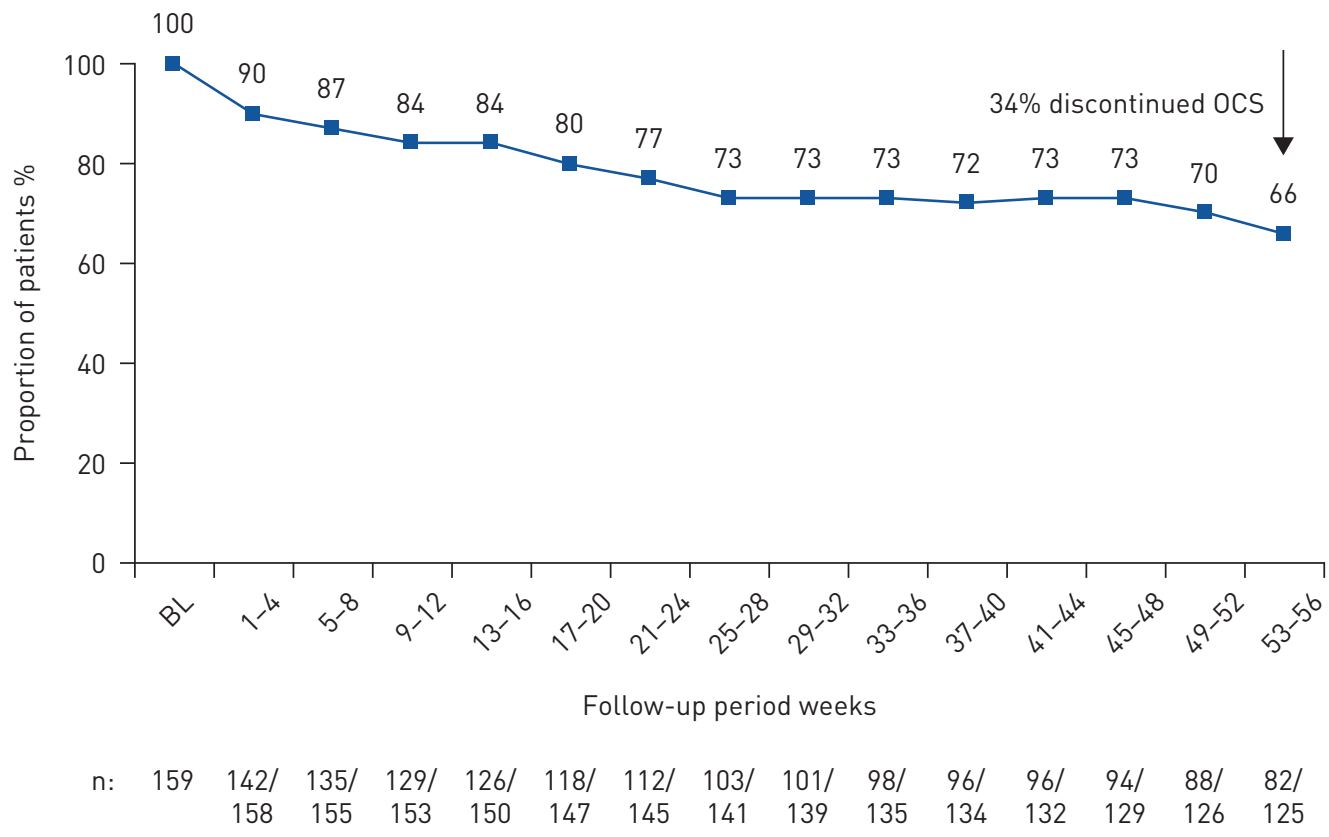

FIGURE 5 Proportion of patients on maintenance oral corticosteroid (OCS) at enrolment who continued with maintenance OCS after treatment initiation with mepolizumab. BL: baseline. Data are from the while-on-treatment estimand for treatment discontinuation (i.e. data considered up to treatment discontinuation). The $\mathrm{n}$-value indicates the number of patients receiving OCS (together with the number of patients with OCS data).

We found the rates of clinically significant exacerbations and exacerbations requiring hospitalisation and/or emergency department visit were significantly reduced with mepolizumab treatment initiation versus before initiation. Reductions in clinically significant exacerbations were observed regardless of older age, maintenance OCS at enrolment or prior use of omalizumab. The treatment policy estimand included data from patients who discontinued mepolizumab, providing a conservative effectiveness estimate. Our results support those from the clinical trials MENSA (ClinicalTrials.gov identifier NCT01691521) and MUSCA (ClinicalTrials.gov identifier NCT02281318), where patients receiving mepolizumab (subcutaneous dose) experienced respective $53 \%$ and $58 \%$ reductions in the rate of clinically significant exacerbations versus placebo, despite the placebo effect observed in both studies [14, 15]. Additionally, our data are also consistent with findings from several smaller, observational studies of real-world mepolizumab treatment for severe asthma [23-27].

$\begin{array}{lc}\text { TABLE } 3 \text { Treatment-related adverse events and serious adverse events } & 368 \\ \text { Total population } & 53(14) \\ \text { Patients with adverse events } & 26(7) \\ \text { Adverse events occurring most frequently ( } \geqslant \mathbf{2 \%} \text { of patients) } & 12(3) \\ \quad \text { Nervous system disorders } & 9(2) \\ \text { General disorders and administration site conditions } & 9(2) \\ \text { Musculoskeletal and connective tissue disorders } & 6(2) \\ \text { Skin and subcutaneous tissue disorders } & 9(2) \\ \text { Gastrointestinal disorders } & 7(2) \\ \text { Patients with adverse events leading to treatment discontinuation } & 2(<1) \\ \text { Patients with adverse events leading to study withdrawal } & 1(<1) \\ \text { Serious adverse events } & 1(<1) \\ \text { Hypersensitivity } & 0(0) \\ \text { Pharyngeal swelling } & \end{array}$

Data are presented as $\mathrm{n}$ or $\mathrm{n}(\%) .{ }^{*}$ : the adverse events leading to treatment discontinuation included headache $(n=2 \quad(<1 \%))$, dizziness $(n=1 \quad(<1 \%))$, paraesthesia $(n=1 \quad<1 \%))$, dyspepsia $(n=1 \quad(<1 \%))$, nausea $(n=1$ $(<1 \%))$, palpitations $(n=1 \quad(<1 \%))$, tachycardia $(n=1 \quad(<1 \%))$, vertigo $(n=1 \quad(<1 \%))$, noncardiac chest pain $(n=1$ $(<1 \%))$, hypersensitivity $(n=1 \quad(<1 \%))$, arthralgia $(n=1 \quad(<1 \%))$, pharyngeal swelling $(n=1 \quad<1 \%))$ and pruritus $(n=1(<1 \%)) ;$ two adverse events were not coded. 
Daily maintenance OCS use was also assessed. We observed a clinically meaningful reduction in the median daily maintenance OCS dose during follow-up in patients who were on maintenance OCS pre-mepolizumab treatment. Reductions were also meaningful when assessed by baseline blood eosinophil count, although the smaller sample sizes resulted in larger variability. Approximately one-third of patients discontinued maintenance OCS by week 53-56. Similar to our results, a 50\% reduction in the median OCS dose in patients receiving mepolizumab versus placebo was demonstrated in the SIRIUS trial (ClinicalTrials.gov identifier NCT01691508) and has also been reported in smaller real-world observational studies [13, 23-27]. These reductions are particularly important in patients with severe asthma given the risk of adverse events associated with chronic SCS use, irrespective of dose level, and the additional healthcare costs related to corticosteroid-induced adverse events in patients with severe asthma $[28,29]$. Thus, this large international, prospective REALITI-A study, together with the findings from these smaller observational studies, provides evidence that the clinical benefits observed with mepolizumab in clinical trials translate to the real-world setting and indicates that mepolizumab may help reduce the severe asthma healthcare burden.

In contrast to RCTs with selected, homogeneous populations, this real-world study included a heterogeneous population, with a broader spectrum of comorbidities and concomitant medications versus those typically permitted in RCTs. Additionally, unlike RCTs, this real-world population was subject to payer reimbursement criteria, which differed among countries. Data from REALITI-A complement those from RCTs; however, more importantly, they highlight mepolizumab effectiveness in the context of real-world clinical practice. The REALITI-A patient population had particularly severe asthma, as approximately half of the patients received maintenance OCS pre-mepolizumab treatment, and patients had an average of 1.2 exacerbations requiring hospitalisation and/or emergency department visit in the 12 months pre-study enrolment. It is perhaps not surprising these early treatment initiators in this analysis had such severe disease as new treatments are often channelled to those in the most severe spectrum of the disease [30]. Furthermore, nearly $50 \%$ of patients in this initial analysis were from the UK, where treatment eligibility criteria are particularly more stringent than enrolment criteria applied in clinical trials and the eligibility criteria in many other REALITI-A countries. However, despite the REALITI-A population in this analysis being more severe than those patients with severe eosinophilic asthma in the RCTs [13-16] with no previous smoking history or lung function status restrictions, clinical outcomes are at least as good as those in the clinical trial setting. Thus, the REALITI-A study provides conformational validity of the more formal trials and, as such, identifies that the therapeutic benefit of mepolizumab is translated in the real-world environment.

The relationship between higher blood eosinophil counts and mepolizumab responses in patients with severe asthma has been identified in previous studies [13-16], with increasing evidence supporting mepolizumab use in patients with baseline blood eosinophil counts $\geqslant 150$ cells $\mu \mathrm{L}^{-1}$ [31-33]. This population is defined by serious morbidity, which increases with higher eosinophil counts (which act as a predictor of mepolizumab response for exacerbation reduction) [15, 34-38]. In our study, similar reductions in the clinically significant exacerbation rate and the median daily maintenance OCS use between pre-treatment and follow-up were seen across all baseline blood eosinophil subgroups, including the $<150$ cells. $\mu \mathrm{L}^{-1}$ subgroup. Although mepolizumab is licensed for the treatment of severe eosinophilic asthma and usually requires evidence of elevated blood eosinophils [18], the treated severe asthma population in this real-world setting included 51 (14\%) patients with baseline blood eosinophil counts $<150$ cells $\mu \mathrm{L}^{-1}$ on entry. However, most of these patients had confirmatory evidence of severe eosinophilic asthma as they had blood eosinophil counts $\geqslant 300$ cells $\mu \mathrm{L}^{-1}$ during the previous year (and run-in period if relevant) (67\% (34 out of 51)) and/or were requiring maintenance OCS at enrolment (63\% (32 out of 51)).

Limitations of real-world studies include the capturing of data from standard clinical care recording; therefore, there may be missing information. To mitigate this, one study entry criterion was that patients needed 12 months of relevant medical records available before enrolment; this was done so information on exacerbation history could be obtained as accurately as possible. Nevertheless, it is possible some historical exacerbations were overlooked, owing to inadequate recording or self-medication, which would lead to an underestimation of the impact of mepolizumab. Additionally, as REALITI-A is a real-world study, therapy could be discontinued or changed by the patient or physician. Patient behaviour may be harder to control in real-world studies and discontinuation rates in real-world studies have been shown to be higher than those observed in RCTs, with patient decision and lack of efficacy accounting for most treatment withdrawals [39]. In this 1-year study, $19 \%$ of patients discontinued treatment, which is similar to the rate observed in other real-world studies [39]. Some of these individuals may not have had a positive response to mepolizumab and discontinued from the study. It is feasible that some patients had severe eosinophilic asthma alongside other diseases, and any continued symptoms in these patients may have been due to nonasthma disease and incorrectly interpreted as a lack of response to mepolizumab. However, only $4 \%$ 
discontinued mepolizumab because of lack of efficacy, so this is unlikely to be a significant confounding factor. Patients may also have withdrawn owing to failure to meet payer reimbursement criteria, an option that was not listed on the electronic case report form. Furthermore, the treatment policy estimand approach for treatment discontinuation used, whereby all who received one mepolizumab dose or more were included, attempts to limit any bias owing to study dropouts. As this is an ongoing study and an interim analysis on a subcohort of patients, changes to the datasets may occur; however, all efforts were made to finalise the data to the best standard. Finally, REALITI-A is not a placebo-controlled study; therefore, outcomes may represent a combination of treatment effect and other behavioural changes. While this is inherent in all real-world open-label studies, this analysis of early initiators in the real world shows that the effects of mepolizumab are consistent with those demonstrated in the severe asthma clinical trials and identifies the clinical benefits of mepolizumab are translated in the severe asthma population in the real world.

In conclusion, data from this initial analysis of mepolizumab treatment initiation in patients with asthma treated in routine clinical practice demonstrated mepolizumab was associated with significant reductions in asthma exacerbations and clinically significant reductions in maintenance OCS use. Additionally, mepolizumab was well tolerated with a safety profile that appeared to be similar to previous clinical studies conducted in patients with severe eosinophilic asthma [14-16]. These data also show the mepolizumab efficacy found in clinical trials translates to the real world and provide valuable insights into treatment outcomes in patients treated in this setting.

Acknowledgements: The authors would like to thank all centres involved in the current study, and Sally Worsley (GSK, Stevenage, UK) for leading study conception and design, and Elaine Irving (GSK, Stevenage, UK), Necdet Gunsoy (GSK, Brentford, UK), Rafael Alfonso (GSK, Collegeville, PA, USA), Namhee Kwon (GSK, Brentford, UK) and Frank Albers (GSK, Research Triangle Park, NC, USA), for their support with study conception and design. Editorial support (in the form of writing assistance, including development of the initial draft from the study report, assembling tables and figures, collating author comments, grammatical editing, and referencing) was provided by Elizabeth Hutchison and Roisin McCorkell (Fishawack Indicia Ltd, Knutsford, UK) and was funded by GSK.

Author contributions: K. Gemzoe and M.K. Van Dyke contributed to the conception and design of the study, and T. Harrison, G.W. Canonica, G. Chupp, J. Lee, F. Schleich, T. Welte and A. Valero contributed to the acquisition of data. All authors contributed to the interpretation of the data, revised the manuscript critically for important intellectual content, gave final approval of the version to be published and agreed to be accountable for all aspects of the work. All authors had access to the study data.

Conflict of interest: T. Harrison reports that the study and writing support was funded by GSK, and received personal fees for lectures and advisory board work from GSK and AstraZeneca, personal fees for advisory board work from Vectura, outside the submitted work. G.W. Canonica reports that the study and writing support was funded by GSK, and received grants and personal fees for lectures and advisory board work from GSK, AstraZeneca, Sanofi-Genzyme, Regeneron and Novartis, outside the submitted work. G. Chupp reports that the study and writing support was funded by GSK, and received grants and personal fees for lectures and advisory board work from GSK, AstraZeneca, Genentech, Sanofi-Genzyme, Regeneron, Teva and Novartis, outside the submitted work. J. Lee reports that the study and writing support was funded by GSK, and received grants from Regeneron, Genentech, Roche and Takeda, grants and personal fees for lectures from GSK, Sanofi-Genzyme, Novartis, Medexus and AstraZeneca, personal fees for lectures from Mylan, Aralez and Merck, outside the submitted work. F. Schleich reports that the study and writing support was funded by GSK, and received grants and personal fees for lectures and advisory board work from AstraZeneca, grants, personal fees for lectures and advisory board work, and nonfinancial support for travel from Chiesi and Novartis, personal fees for lectures from Menarini and Mundipharma, grants and personal fees for lectures, consultancy and advisory board work from GSK, outside the submitted work. T. Welte reports that the study and writing support was funded by GSK, and received grants and personal fees for lectures and advisory board work from AstraZeneca, personal fees for lectures and advisory board work from Novartis and Sanofi, personal fees for advisory board work from GSK, outside the submitted work. A. Valero reports that the study and writing support was funded by GSK, and received personal fees for consultancy and lectures from AstraZeneca, Novartis and Mundipharma, personal fees for consultancy from Sanofi and Boehringer, personal fees for lectures from Chiesi and GSK, outside the submitted work. K. Gemzoe reports that the study and writing support was funded by GSK, and is an employee of and holds shares/options in GSK. A. Maxwell reports that the study and writing support was funded by GSK, and is an employee of and holds shares/options in GSK. S. Joksaite reports that the study and writing support was funded by GSK, and is an employee of and holds shares/options in GSK. S. Yang reports that the study and writing support was funded by GSK, and is an employee of and holds shares/options in GSK. P. Howarth reports that the study and writing support was funded by GSK, and is an employee of and holds shares/options in GSK. M.K. Van Dyke reports that the study and writing support was funded by GSK, and is an employee of and holds shares/options in GSK.

Support statement: This study was funded by GSK (GSK study identifier 204710). Funding information for this article has been deposited with the Crossref Funder Registry.

\section{References}

1 Chung KF, Wenzel SE, Brozek JL, et al. International ERS/ATS guidelines on definition, evaluation and treatment of severe asthma. Eur Respir J 2014; 43: 343-373. 
2 The Global Asthma Network. The Global Asthma Report 2018. 2018. www.globalasthmareport.org/Global\% 20Asthma\%20Report\%202018.pdf Date last accessed: September 14, 2019.

3 Foster JM, McDonald VM, Guo M, et al. "I have lost in every facet of my life": the hidden burden of severe asthma. Eur Respir J 2017; 50: 1700765.

4 Chastek B, Korrer S, Nagar SP, et al. Economic burden of illness among patients with severe asthma in a managed care setting. J Manag Care Spec Pharm 2016; 22: 848-861.

5 Kerkhof M, Tran TN, Soriano JB, et al. Healthcare resource use and costs of severe, uncontrolled eosinophilic asthma in the UK general population. Thorax 2018; 73: 116-124.

6 Zeiger RS, Schatz M, Dalal AA, et al. Utilization and costs of severe uncontrolled asthma in a managed-care setting. J Allergy Clin Immunol Pract 2016; 4: 120-129.

7 Buhl R, Humbert M, Bjermer L, et al. Severe eosinophilic asthma: a roadmap to consensus. Eur Respir J 2017; 49: 1700634 .

8 Brown T, Jones T, Gove K, et al. Randomised controlled trials in severe asthma: selection by phenotype or stereotype. Eur Respir J 2018; 52: 1801444.

9 Schleich F, Brusselle G, Louis R, et al. Heterogeneity of phenotypes in severe asthmatics. The Belgian Severe Asthma Registry (BSAR). Respir Med 2014; 108: 1723-1732.

10 Brusselle GG, Maes T, Bracke KR. Eosinophils in the spotlight: eosinophilic airway inflammation in nonallergic asthma. Nat Med 2013; 19: 977-979.

11 Wenzel S. Severe asthma: from characteristics to phenotypes to endotypes. Clin Exp Allergy 2012; 42: 650-658.

12 Menzella F, Lusuardi M, Galeone C, et al. Profile of anti-IL-5 mAb mepolizumab in the treatment of severe refractory asthma and hypereosinophilic diseases. J Asthma Allergy 2015; 8: 105-114.

13 Bel EH, Wenzel SE, Thompson PJ, et al. Oral glucocorticoid-sparing effect of mepolizumab in eosinophilic asthma. N Engl J Med 2014; 371: 1189-1197.

14 Chupp GL, Bradford ES, Albers FC, et al. Efficacy of mepolizumab add-on therapy on health-related quality of life and markers of asthma control in severe eosinophilic asthma (MUSCA): a randomised, double-blind, placebo-controlled, parallel-group, multicentre, phase 3b trial. Lancet Respir Med 2017; 5: 390-400.

15 Ortega HG, Liu MC, Pavord ID, et al. Mepolizumab treatment in patients with severe eosinophilic asthma. $N$ Engl J Med 2014; 371: 1198-1207.

16 Pavord ID, Korn S, Howarth P, et al. Mepolizumab for severe eosinophilic asthma (DREAM): a multicentre, double-blind, placebo-controlled trial. Lancet 2012; 380: 651-659.

17 European Medicines Agency. Assessment Report - Nucala. 2018. www.ema.europa.eu/en/documents/ variation-report/nucala-h-c-3860-ii-0013-g-epar-assessment-report-variation_en.pdf Date last accessed: August 1, 2019.

18 GlaxoSmithKline. Mepolizumab (NUCALA) US prescribing information. 2019. https:/gsksource.com/pharma/ content/dam/GlaxoSmithKline/US/en/Prescribing_Information/Nucala/pdf/NUCALA-PI-PIL.PDF Date last accessed: November 21, 2019.

19 Pahus L, Jaffuel D, Vachier I, et al. Randomised controlled trials in severe asthma: selection by phenotype or stereotype. Eur Respir J 2019; 53: 1801444.

20 Saturni S, Bellini F, Braido F, et al. Randomized controlled trials and real life studies. Approaches and methodologies: a clinical point of view. Pulm Pharmacol Ther 2014; 27: 129-138.

21 Roche N, Anzueto A, Bosnic Anticevich S, et al. The importance of real-life research in respiratory medicine: manifesto of the Respiratory Effectiveness Group: endorsed by the International Primary Care Respiratory Group and the World Allergy Organization. Eur Respir J 2019; 54: 1901511.

22 Hahn G, Meeker W. Statistical Intervals: A Guide for Practitioners. New York, Wiley, 1991.

23 Bagnasco D, Caminati M, Menzella F, et al. One year of mepolizumab. Efficacy and safety in real-life in Italy. Pulm Pharmacol Ther 2019; 58: 101836.

24 Pertzov B, Unterman A, Shtraichman O, et al. Efficacy and safety of mepolizumab in a real-world cohort of patients with severe eosinophilic asthma. J Asthma 2019; in press [https://doi.org/10.1080/02770903.2019.1658208].

25 Montero-Perez O, Contreras-Rey MB, Sanchez-Gomez E. Effectiveness and safety of mepolizumab in severe refractory eosinophilic asthma: results in clinical practice. Drugs Context 2019; 8: 212584.

26 Pelaia C, Busceti MT, Solinas S, et al. Real-life evaluation of the clinical, functional, and hematological effects of mepolizumab in patients with severe eosinophilic asthma: results of a single-centre observational study. Pulm Pharmacol Ther 2018; 53: 1-5.

27 Strauss RA, Jawhari N. Mepolizumab in the treatment of severe eosinophilic asthma: results from a physician in the field. Ann Allergy Asthma Immunol 2018; 121: 121-123.

28 Canonica GW, Colombo GL, Bruno GM, et al. Shadow cost of oral corticosteroids-related adverse events: a pharmacoeconomic evaluation applied to real-life data from the Severe Asthma Network in Italy (SANI) registry. World Allergy Organ J 2019; 12: 100007.

29 Lefebvre P, Duh MS, Lafeuille MH, et al. Burden of systemic glucocorticoid-related complications in severe asthma. Curr Med Res Opin 2017; 33: 57-65.

30 Petri H, Urquhart J. Channeling bias in the interpretation of drug effects. Stat Med 1991; 10: 577-581.

31 Yancey SW, Bradford ES, Keene ON. Disease burden and efficacy of mepolizumab in patients with severe asthma and blood eosinophil counts of $\geqslant 150-300$ cells/ $\mu \mathrm{L}$. Respir Med 2019; 151: 139-141.

32 Yancey SW, Keene ON, Albers FC, et al. Biomarkers for severe eosinophilic asthma. J Allergy Clin Immunol 2017; 140: 1509-1518.

33 Holguin F, Cardet JC, Chung KF, et al. Management of severe asthma: a European Respiratory Society/American Thoracic Society guideline. Eur Respir J 2020; 55: 1900588.

34 Zeiger RS, Schatz M, Li Q, et al. High blood eosinophil count is a risk factor for future asthma exacerbations in adult persistent asthma. J Allergy Clin Immunol Pract 2014; 2: 741-750.

35 Makela MJ, Christensen HN, Karlsson A, et al. Health care resource utilization and characteristics of patients with eosinophilic asthma in secondary health care in Finland. Eur Clin Respir J 2018; 5: 1458560.

36 Price D, Wilson AM, Chisholm A, et al. Predicting frequent asthma exacerbations using blood eosinophil count and other patient data routinely available in clinical practice. J Asthma Allergy 2016; 9: 1-12. 
37 Albers FC, Licskai C, Chanez P, et al. Baseline blood eosinophil count as a predictor of treatment response to the licensed dose of mepolizumab in severe eosinophilic asthma. Respir Med 2019; 159: 105806.

38 Ortega HG, Yancey SW, Mayer B, et al. Severe eosinophilic asthma treated with mepolizumab stratified by baseline eosinophil thresholds: a secondary analysis of the DREAM and MENSA studies. Lancet Respir Med 2016; 4: 549-556.

39 Caminati M, Senna G, Stefanizzi G, et al. Drop-out rate among patients treated with omalizumab for severe asthma: literature review and real-life experience. BMC Pulm Med 2016; 16: 128. 